European Journal of

Organic Chemistry
Postfach 101161 69451 Weinheim

Germany

Courier services:

Boschstraße 12

69469 Weinheim

Germany

Tel.: (+49) 6201606255

Fax: (+49) 6201606203

E-mail: eurjoc@wiley-vch.de

Manuscript No.

\section{Reprints/lssues}

You are entitled to a PDF for 25 hardcopies of your article. You also have the opportunity to order further issues, reprints, posters or a PDF for an unlimited number of hardcopies at the quoted rates.

Reprints of European Journal of Organic Chemistry articles are very popular. Whole issues, reprints, posters and high-quality PDFs are available at the rates given on a separate sheet. There is no surcharge for color reprints.

For overseas orders please note that you will receive your issues, reprints or posters by airmail unless you specifically opt for receiving them by surface mail. An appropriate surcharge will be levied to cover the higher postal rates.

Please bill me for:

\section{Reprints}

$$
\text { (quantity) }
$$

\section{Issues}

$$
\text { (quantity) }
$$

and send them by

$\square$ surface mail $\square$ courier service

\section{Cover Posters}

Posters are available of all the published covers in two sizes (see attached price list).

DIN A2 (42 x $60 \mathrm{~cm} / 17 \times 24 \mathrm{in})$ :

$\square$ front cover $\square$ back cover

DIN A1 (60 x 84 cm/ 24 x 33in):

$\square$ front cover $\square$ back cover

\section{PDF (unlimited number of hardcopies)}

\section{Please bill me for}

$\square$ a PDF file (high resolution)

\section{E-mail address}

Please note that posting of the final published version on the open internet is not permitted but it can be forwarded to colleagues, added to promotion packages, etc.

$\star$ Special Offer $\star$ If you order 200 or more reprints you will get a PDF file for half-price. reprints and a PDF file

Mail reprints/ issues/ posters to (no P.O. Boxes)

\section{Terms of Payment}

\section{Send INVOICE to}

\section{VAT number}

Tax-free charging can only be processed with the VAT number of the institute/company. To prevent delays with the processing, please provide us with the VAT number with this order.

\section{Purchase Order No.:}

\section{Charge the CREDIT CARD}

We accept

\section{VISA}
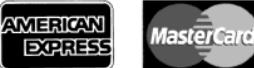

For your security please create a secured Credit Card Token which is a combination of numbers and letters that is used instead of the credit card information. Click here:

https://www.wiley-vch.de/editorial production/index.php

TOKEN

Date, Signature 


\section{WILEY-VCH}

\section{Price List (2015)}

The prices listed below are valid only for orders received in the course of 2015. Minimum order is 50 copies. Delivery time will be approximately 3 weeks after the date of publication.

If more than 500 copies are ordered, special prices are available upon request. Single issues are available to authors at a reduced price. The prices include mailing and handling charges (with the exception of the additional costs incurred for airmail delivery and courier services). The prices are exclusive of VAT.

Reprints, posters, and issues for overseas orders are shipped by airmail ( 25.00 Euro surcharge). If you would like to receive them by surface mail please indicate this on the accompanying order form (postage for shipping posters within Europe: 15.00 Euro). If you would like to use a courier service please indicate this on the order form. The cost for the courier service is 75.00 Euro.

\section{Information regarding VAT}

The charges for Reprints, Issues or Posters are considered to be "supply of goods" and therefore subject to German VAT. However, if you are an institutional customer outside Germany, the tax can be waived if you provide us with the VAT number of your company. Non-EU customers may have a VAT number starting with "EU" instead of their country code if they are registered with the EU tax authorities. If you do not have an EU VAT number and you are a taxable person doing business in a non-EU country, please provide certification from your local tax authorities confirming that you are a taxable person under local tax law. Please note that the certification must confirm that you are a taxable person and are conducting an economic activity in your country. Note: certifications confirming that you are a tax-exempt legal body (non-profit organization, public body, school, political party, etc.) in your country do not exempt you from paying German VAT.

\begin{tabular}{|c|c|c|c|c|c|c|}
\hline \multirow{2}{*}{$\begin{array}{l}\text { Reprints } \\
\text { Size (pages) }\end{array}$} & \multicolumn{6}{|c|}{ Price for orders (in Euro) } \\
\hline & 50 copies & 100 copies & 150 copies & 200 copies & 300 copies & 500 copies \\
\hline $1-4$ & 345.- & 395.- & 425.- & 445.- & 548.- & 752.- \\
\hline $5-8$ & 490.- & 573.- & 608.- & 636.- & 784.- & 1077.- \\
\hline $9-12$ & 640.- & 739.- & 786.- & 824.- & 1016.- & 1396.- \\
\hline $13-16$ & 780.- & 900.- & 958.- & 1004.- & 1237.- & 1701.- \\
\hline $17-20$ & 930.- & 1070.- & 1138.- & 1196.- & 1489.- & 2022.- \\
\hline $\begin{array}{l}\text { for every additional } \\
4 \text { pages }\end{array}$ & 147.- & 169.- & 175.- & 188.- & 231.- & 315.- \\
\hline Issues & \multicolumn{2}{|c|}{22 Euro (1 copy) } & \multicolumn{2}{|c|}{ PDF (high resolution) } & 330 Euro & \\
\hline Cover Posters & $\begin{array}{l}\text { - DIN A2 (42 } \\
\text { - DIN A1 (60 }\end{array}$ & $\begin{array}{l}\text { m/ } 17 \text { x 24in): } \\
\text { m/ } 24 \text { x 33in): }\end{array}$ & $\begin{array}{l}38 \text { Euro } \\
48 \text { Euro }\end{array}$ & & & \\
\hline
\end{tabular}

\section{$\star$ Special Offer $\star$}

If you order 200 or more reprints you will get a PDF (high resolution) for half-price. 


\title{
Gold- and Silver-Catalyzed 7-endo-dig Cyclizations for the Synthesis of Oxazepines
}

\author{
Anatoly A. Peshkov, ${ }^{[a]}$ Anton A. Nechaev, ${ }^{[a]}$ Olga P. Pereshivko, ${ }^{[b]}$ Jan L. Goeman, ${ }^{[c]}$ \\ Johan Van der Eycken, ${ }^{[c]}$ Vsevolod A. Peshkov, ${ }^{*[b]}$ and Erik V. Van der Eycken*[a]
}

Keywords: Synthetic methods / Multicomponent reactions / Medium-ring compounds / Cyclization / Nitrogen heterocycles merizations of hydroxypropargylamides into oxazepines is described. Three different types of hydroxypropargylamide substrates derived from either amide coupling or Ugi reactions have been validated and provide selective and general access to a medium-ring oxazepine core.

\section{Introduction}

The activation of triple bonds by coinage metals salts towards intramolecular nucleophilic attack resulting in hetero- or carbocyclization has become one of the prevalent strategies in modern heterocyclic chemistry. ${ }^{[1]}$ Such processes not only provide an efficient entry to many common heterocycles ${ }^{[2]}$ but also broaden the existing chemical space by leading to a large variety of hitherto unknown and structurally complex heterocyclic scaffolds, ${ }^{[3]}$ which could be of great interest in medicinal chemistry. The procedures for the synthesis of medium-ring heterocycles ${ }^{[4]}$ are of particular importance owing to the widespread occurrence of such products in natural and biologically active compounds. Our group has recently contributed to this area by applying cationic gold catalysis to the synthesis of azocino[5,4-b]indoles, ${ }^{[5]}$ azepino- and azocino[ $\left.5,4,3-c, d\right]$ indoles, ${ }^{[6]}$ benzo[b]pyrrolo[1,2-i] $[1,5]$ diazonines, ${ }^{[7]}$ and imidazo[1,4]diazepines. ${ }^{[8]}$

In the course of one of our previous studies devoted to the synthesis of azocino[5,4- $b$ ]indoles 2 through a gold-catalyzed intramolecular triple bond hydroarylation ${ }^{[9]}$ (Scheme 1, a), we accidentally discovered another potentially useful transformation (Scheme 1, b). Although the common pathway involved the carbocyclization of proparg-

[a] Laboratory for Organic \& Microwave-Assisted Chemistry (LOMAC), Department of Chemistry, University of Leuven (KU Leuven),

Celestijnenlaan 200F, 3001 Leuven, Belgium

E-mail: erik.vandereycken@chem.kuleuven.be http://chem.kuleuven.be/en/research/mds/lomac

[b] College of Chemistry, Chemical Engineering and Materials Science, Soochow University,

Dushu Lake Campus, Suzhou 215123, P. R. China E-mail: vsevolod@suda.edu.cn

[c] Laboratory for Organic and Bioorganic Synthesis, Department of Organic and Macromolecular Chemistry, Ghent University, Krijgslaan 281 (S4), 9000 Ghent, Belgium

Supporting information for this article is available on the WWW under http://dx.doi.org/10.1002/ejoc.201500357.

tional group, yielded oxazepine $\mathbf{4}$ as the major product along with minor amounts of morpholine 5. ${ }^{[10]}$ The current work aims to extend the scope of this approach to establish a general and selective entry towards the oxazepine scaffold. ${ }^{[11,12]}$

(a)
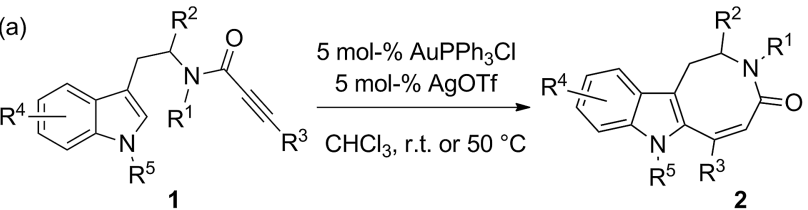

$\mathrm{R}^{1}=\mathrm{Alk} ; \mathrm{R}^{2}=\mathrm{H}, \mathrm{Alk}, \mathrm{COOMe}$ $\mathrm{R}^{3}=\mathrm{Alk}, \mathrm{Ar} ; \mathrm{R}^{4}=\mathrm{H}, 5-\mathrm{MeO} ; \mathrm{R}^{5}=\mathrm{H}, \mathrm{Me}$
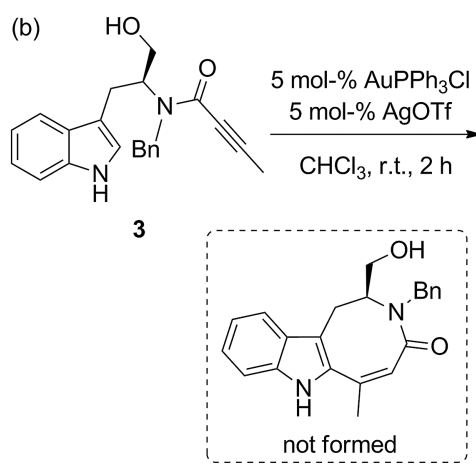<smiles>CC1=CC(=O)N(Cc2ccccc2)CC1Cc1c[nH]c2ccccc12</smiles>

$4(76 \%)$<smiles>C/C=C1\OCC(Cc2c[nH]c3ccccc23)N(Cc2ccccc2)C1=O</smiles>

$5(17 \%)$
Scheme 1. From azocino[5,4-b]indoles 2 towards oxazepines 4.

\section{Results and Discussion}

We started with the synthesis of several hydroxypropargylamides 3 through the direct amide coupling of unprotected secondary amino alcohols with 3-substituted propiolic acids. All reactions proceeded with moderate-to-good efficiency with $N, N^{\prime}$-dicyclohexylcarbodiimide (DCC) as a convenient coupling reagent (Scheme 2). 

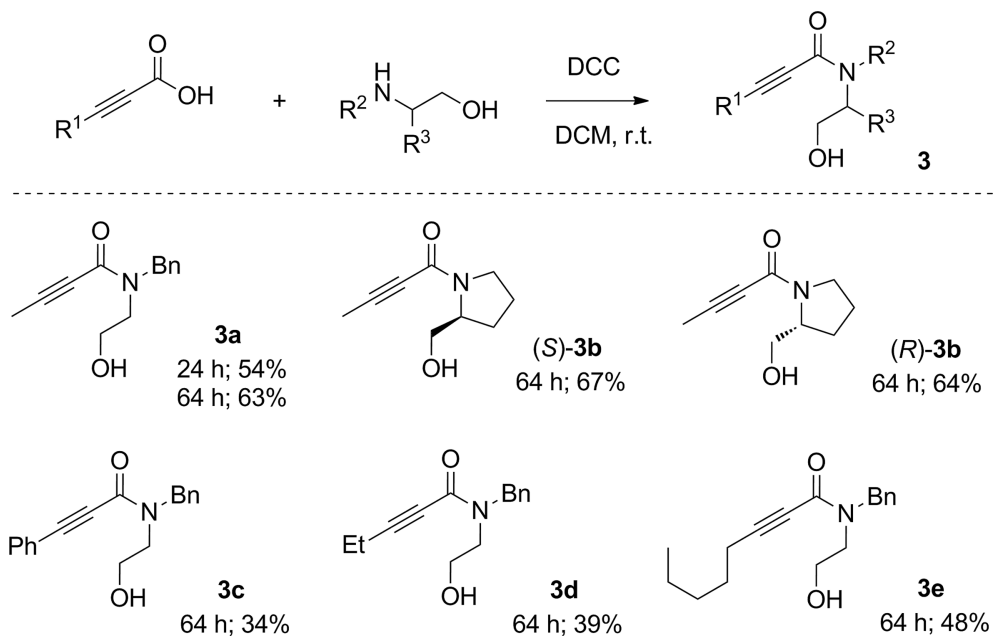

Scheme 2. Synthesis of hydroxypropargylamides 3 through amide coupling reactions.

Another set of hydroxypropargylamide substrates $\mathbf{6}$ and 7 were attained through four-component Ugi reactions, ${ }^{[13,14]}$ which at the same time acted as a potent diversification tool. The incorporation of a hydroxyl function into the amine component yielded hydroxypropargylamides of type 6 (Scheme 3, a). The application of glycolaldehyde dimer provided hydroxypropargylamides of type 7 (Scheme 3, b).

Next, we performed a catalyst screening with hydroxypropargylamide $\mathbf{3 a}$ as a model substrate (Table 1). The application of chloro(triphenylphosphine)gold(I) in combination with silver(I) triflate (AgOTf) to form an in situ catalytically active cationic gold complex led to the full conversion of $3 \mathrm{a}$ within $2 \mathrm{~h}$ at $25^{\circ} \mathrm{C}$ and afforded the desired oxazepine $\mathbf{4 a}$ in $96 \%$ isolated yield (Table 1, Entry 1). Silver triflate alone also catalyzed this transformation (Table 1,

(a)
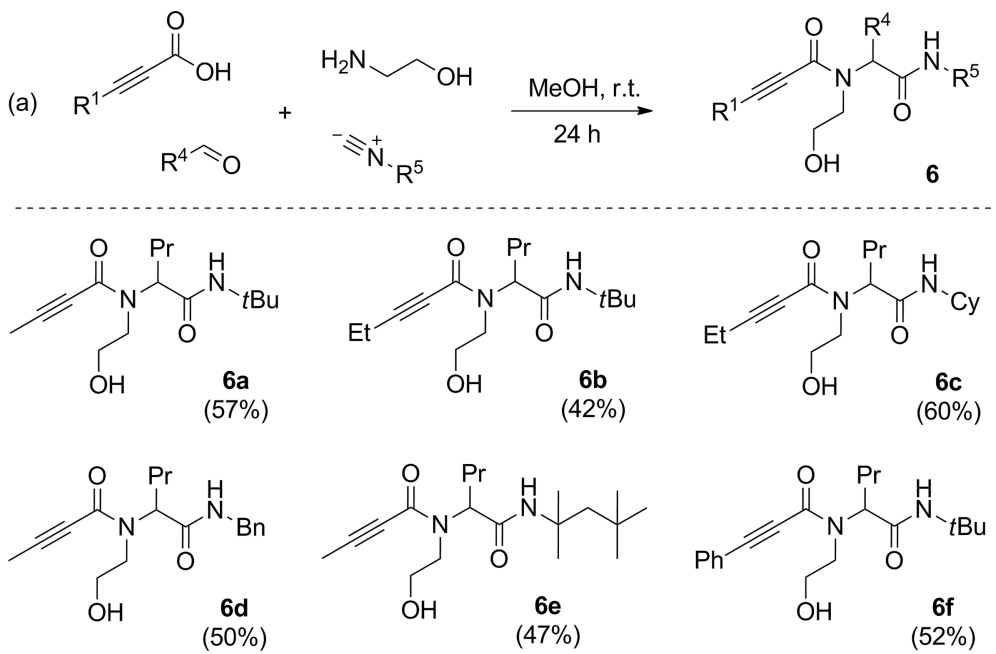<smiles>CC#CC(=O)N(CCO)C(C(=O)NC)C(=O)NC(C)(C)CC(C)(C)C</smiles><smiles>CCCC(C(=O)NCC(C)C)N(CCO)C(=O)C#Cc1ccccc1</smiles><smiles>CC#CC(=O)N(CCO)C(CC(C)C)C(=O)NC(C)(C)C</smiles><smiles>CC#CC(=O)N(CCO)C(Cc1ccccc1)C(=O)NC(C)(C)C</smiles>

(b)<smiles>[R]NCC(C)OC</smiles>

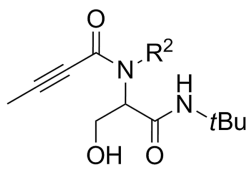

$7 a ; R^{2}=B u, 68 \%$

$7 \mathbf{b} ; R^{2}=P M B, 40 \%$

Scheme 3. Synthesis of hydroxypropargylamides 6 and 7 through Ugi reactions. 
Table 1. Catalyst screening for the cycloisomerization of hydroxypropargylamide $\mathbf{3 a}$ into oxazepine $\mathbf{4 a}$. $^{[a]}$

\begin{tabular}{|c|c|c|c|c|}
\hline Entry & Catalyst & Conditions & Conversion $[\%]^{[\mathrm{b}]}$ & Yield $[\%]^{[\mathrm{c}]}$ \\
\hline 1 (conditions A) & $5 \mathrm{~mol}-\% \mathrm{AuPPh}_{3} \mathrm{Cl} / \mathrm{AgOTf}$ & $2 \mathrm{~h}, 25^{\circ} \mathrm{C}$ & 100 & 96 \\
\hline 2 & 5 mol- $\%$ AgOTf & $24 \mathrm{~h}, 25^{\circ} \mathrm{C}$ & 82 & 78 \\
\hline 3 & 5 mol- $\%$ AgOTf & $2 \mathrm{~h}, 50^{\circ} \mathrm{C}$ & 93 & 85 \\
\hline 4 (conditions B) & 5 mol- $\%$ AgOTf & $15 \mathrm{~h}, 50^{\circ} \mathrm{C}$ & 100 & 91 \\
\hline 5 & $5 \mathrm{~mol}^{-} \% \mathrm{AuPPh}_{3} \mathrm{Cl}$ & $24 \mathrm{~h}, 25^{\circ} \mathrm{C}$ or $3 \mathrm{~h}, 50^{\circ} \mathrm{C}$ & 0 & 0 \\
\hline 6 & $5 \mathrm{~mol}-\% \mathrm{Cu}(\mathrm{OTf})_{2}$ & $24 \mathrm{~h}, 25^{\circ} \mathrm{C}$ or $3 \mathrm{~h}, 50^{\circ} \mathrm{C}$ & 0 & 0 \\
\hline 7 & $20 \mathrm{~mol}-\% \mathrm{TsOH} \cdot \mathrm{H}_{2} \mathrm{O}$ & $15 \mathrm{~h}, 50^{\circ} \mathrm{C}$ & 0 & 0 \\
\hline 8 & $5 \mathrm{~mol}^{-} \% \mathrm{AuCl}_{3}$ & $2 \mathrm{~h}, 25^{\circ} \mathrm{C}$ & 39 & 35 \\
\hline 9 & $5 \mathrm{~mol}^{-} \% \mathrm{AuCl}_{3}$ & $2 \mathrm{~h}, 50^{\circ} \mathrm{C}$ & 47 & 40 \\
\hline 10 & $5 \mathrm{~mol}^{-} \% \mathrm{AuCl}_{3}$ & $24 \mathrm{~h}, 50^{\circ} \mathrm{C}$ & 60 & 51 \\
\hline
\end{tabular}

[a] The reactions were run on $0.3 \mathrm{mmol}$ scale in dry $\mathrm{CHCl}_{3}(1.2 \mathrm{~mL})$. [b] Determined from ${ }^{1} \mathrm{H}$ NMR spectroscopy of a crude reaction mixture. [c] Yield of isolated product.

Entries 2-4), although an extended time and an elevated reaction temperature of $50{ }^{\circ} \mathrm{C}$ were required to achieve full conversion of 3a and provide $\mathbf{4 a}$ in $91 \%$ isolated yield (Table 1, Entry 4). At the same time, chloro(triphenylphosphine)gold(I) alone, copper(II) triflate, and p-toluenesulfonic acid $(\mathrm{TsOH})$ failed to catalyze this process (Table 1,

71 Entries 5-7). With the application of gold(III) chloride, the desired oxazepine product $4 \mathbf{a}$ could be obtained in moderate yield but it was difficult to drive the reaction to completion (Table 1, Entries 8-10). Probably, the reaction slows down owing to the degradation of the catalyst over prolonged reaction times.

With these results in hand, we explored the substrate scope. The cycloisomerizations of all prepared hydroxypropargylamides $3, \mathbf{6}$, and $\mathbf{7}$ were attempted under both cationic gold (conditions A) and silver triflate (conditions B)

81 catalysis, and the target oxazepines $\mathbf{4}$ were delivered in all cases in good-to-high yields with comparable efficiency (Table 2). Importantly, no racemization occurred in the reactions with substrates $(S)$-3b and $(R)$-3b derived from enantiopure prolinols; this demonstrates the mild character of both the gold- and silver-catalyzed protocols (Table 2, Entries 1 and 2). For the silver-catalyzed cyclizations of hydroxypropargylamides $\mathbf{3 c}$ and $\mathbf{6}$ bearing a phenyl group on the triple-bonded atom as well as that of hydroxypropargylamide $\mathbf{7 a}$, an increased catalyst loading was required to reach full conversion (Table 2, Entries 3, 11, and 14). Finally, it is important to stress that all reactions led exclusively to the formation of oxazepine $\mathbf{4}$ by 7-endo-dig cycloisomerization.

\section{Conclusions}

We have successfully established an efficient and selective route for the synthesis of oxazepines through the cycloisomerization of readily accessible hydroxypropargylamides in the presence of a gold or silver catalyst. The scope of the process has been thoroughly explored with various types of substrates, which resulted in the generation of a small yet diverse library of target compounds.

\section{Experimental Section}

General Information: ${ }^{1} \mathrm{H}$ and ${ }^{13} \mathrm{C}$ NMR spectra were recorded at 300 and $75 \mathrm{MHz}$, respectively, with a Bruker Avance instrument. The ${ }^{1} \mathrm{H}$ and ${ }^{13} \mathrm{C}$ NMR chemical shifts are reported in ppm relative to tetramethylsilane, and the residual solvent signal was used as the internal reference. High-resolution EI mass spectra were recorded with a Kratos MS50TC system with a resolution of 10000. The ion-source temperature was $150-250{ }^{\circ} \mathrm{C}$ as required. High-resolution ESI mass spectra were recorded with an Agilent 1100 series HPLC instrument coupled to an Agilent 6220A TOF-MSD spectrometer equipped with an ESI/atmospheric pressure chemical ionization (ESI/APCI) source. Specific rotations were measured with a PolAAr 20 automatic polarimeter.

General Procedure for the Synthesis of Hydroxypropargylamides 3 through Amide Coupling Reactions: 3-Substituted propiolic acid $(2.2 \mathrm{mmol})$ was dissolved in dry dichloromethane (DCM, $10 \mathrm{~mL})$, and the appropriate aminoethanol $(2 \mathrm{mmol})$ and DCC $(2.3 \mathrm{mmol})$ were added. The resulting mixture was stirred at room temp. for $64 \mathrm{~h}$ in a sealed screw-cap vial. The subsequently formed precipitate of $N, N^{\prime}$-dicyclohexylurea was removed by filtration and washed with DCM. The combined organic layers were concentrated and subjected to silica gel column chromatography.

$\mathrm{N}$-Benzyl- $\mathrm{N}$-(2-hydroxyethyl)but-2-ynamide (3a): Elution with heptane/EtOAc $(50 \rightarrow 100 \%)$ delivered pure $3 \mathbf{a}$ as a ca. 3:7 mixture of rotamers, yield $274 \mathrm{mg}, 63 \% .{ }^{1} \mathrm{H}$ NMR $\left(300 \mathrm{MHz}, \mathrm{CDCl}_{3}\right): \delta=$ 7.45-7.15 (m, $5 \mathrm{H}), 4.87(\mathrm{~s}, 1.4 \mathrm{H}), 4.71(\mathrm{~s}, 0.6 \mathrm{H}), 3.82-3.57(\mathrm{~m}$, $2.6 \mathrm{H}), 3.54-3.07(\mathrm{~m}, 2.4 \mathrm{H}), 2.08-1.93(\mathrm{~m}, 3 \mathrm{H}) \mathrm{ppm} .{ }^{13} \mathrm{C} \mathrm{NMR}$ $\left(75 \mathrm{MHz}, \mathrm{CDCl}_{3}\right): \delta=156.3,155.5,136.8,136.3,128.9,128.7$, $128.04,127.96,127.5,90.3,89.9,73.3,73.2,60.9,60.5,54.0,50.2$, $48.2,47.4,4.1$ ppm. HRMS (EI): calcd. for $\mathrm{C}_{13} \mathrm{H}_{15} \mathrm{NO}_{2}[\mathrm{M}]^{+}$ 217.1103; found 217.1118.

(S)-1-[2-(Hydroxymethyl)pyrrolidin-1-yl]but-2-yn-1-one [(S)-3b]: Elution with pure EtOAc delivered pure $(S)-3 \mathbf{b}$ as a ca. 1:4 mixture of rotamers, yield $224 \mathrm{mg}, 67 \% .{ }^{1} \mathrm{H}$ NMR $\left(300 \mathrm{MHz}, \mathrm{CDCl}_{3}\right): \delta=$ 4.70 (br. d, $J=6.1 \mathrm{~Hz}, 0.8 \mathrm{H}), 4.27-4.05(\mathrm{~m}, 1 \mathrm{H}), 3.94-3.81(\mathrm{~m}$, $0.8 \mathrm{H}), 3.80-3.50(\mathrm{~m}, 3 \mathrm{H}), 3.50-3.37(\mathrm{~m}, 0.2 \mathrm{H}), 2.98$ (br. $\mathrm{s}, 0.2$
101 
Table 2. Cycloisomerizations of hydroxypropargylamide precursors $\mathbf{3}, \mathbf{6}$, and $\mathbf{7}$ into oxazepines 4 . $^{[a]}$

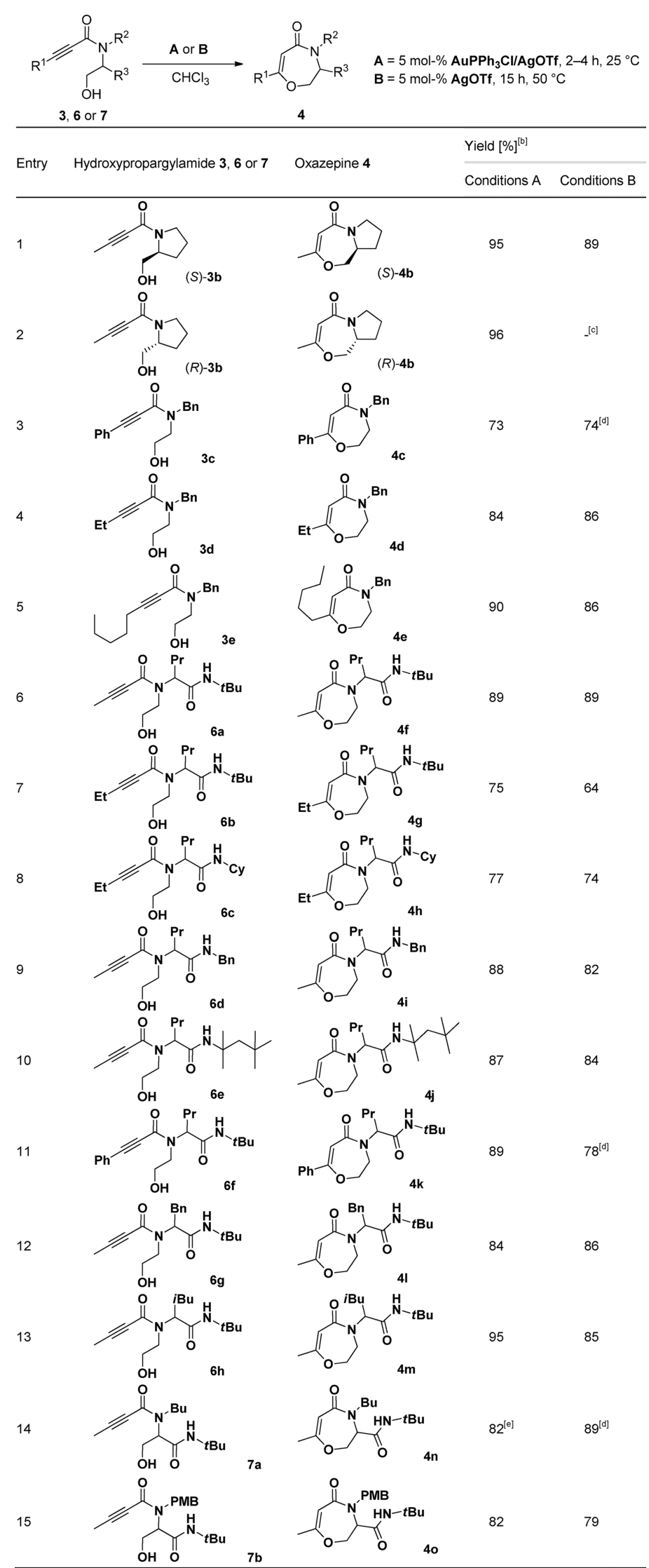

[a] The reactions were run on $0.3 \mathrm{mmol}$ scale in dry $\mathrm{CHCl}_{3}(1.2 \mathrm{~mL})$. [b] Yield of isolated product. [c] The reaction was not performed. [d] $20 \mathrm{~mol}-\%$ of AgOTf was used, and the reaction was run for $20 \mathrm{~h}$. [e] The reaction was run for $16 \mathrm{~h}$. 
$\mathrm{H}), 2.20-1.75(\mathrm{~m}, 6.2 \mathrm{H}), 1.74-1.58(\mathrm{~m}, 0.8 \mathrm{H}) \mathrm{ppm} .{ }^{13} \mathrm{C}$ NMR $\left(75 \mathrm{MHz}, \mathrm{CDCl}_{3}\right): \delta=155.1,153.2,89.7,87.4,74.4,74.1,66.4$, 64.0, 61.1, 60.4, 49.7, 46.8, 28.7, 27.7, 23.9, 22.6, 4.00, $3.97 \mathrm{ppm}$. HRMS (EI): calcd. for $\mathrm{C}_{9} \mathrm{H}_{13} \mathrm{NO}_{2}[\mathrm{M}]^{+}$167.0946; found 167.0952.

$(R)-1-[2-(H y d r o x y m e t h y l) p y r r o l i d i n-1-y l] b u t-2-y n-1-o n e \quad[(R)-3 b]:$ Elution with pure EtOAc delivered pure $(R)-3 \mathbf{b}$ as a ca. 1:4 mixture of rotamers, yield $214 \mathrm{mg}, 64 \%$.

$146 \mathrm{~N}$-Benzyl- $\mathrm{N}$-(2-hydroxyethyl)-3-phenylpropiolamide (3c): Elution with $\mathrm{DCM} / \mathrm{Et}_{2} \mathrm{O}(20 \%)$ delivered pure $3 \mathbf{c}$ as a ca. 3:7 mixture of rotamers, yield $190 \mathrm{mg}, 34 \% .{ }^{1} \mathrm{H}$ NMR $\left(300 \mathrm{MHz}, \mathrm{CDCl}_{3}\right): \delta=$ 7.59-7.27 (m, $10 \mathrm{H}), 4.96(\mathrm{~s}, 1.4 \mathrm{H}), 4.78(\mathrm{~s}, 0.6 \mathrm{H}), 3.87-3.80(\mathrm{~m}$, $0.6 \mathrm{H}), 3.79-3.70(\mathrm{~m}, 2 \mathrm{H}), 3.60-3.53(\mathrm{~m}, 1.4 \mathrm{H}) \mathrm{ppm} .{ }^{13} \mathrm{C} \mathrm{NMR}$ $\left(75 \mathrm{MHz}, \mathrm{CDCl}_{3}\right): \delta=156.2,155.3,136.7,136.2,132.5,132.4$, $130.3,130.1,128.9,128.7,128.55,128.52,128.14,128.06,127.64$, $127.58,120.4,120.2,91.3,90.9,81.6,81.5,61.2,60.8,54.3,50.5$, 48.6, 47.8 ppm. HRMS (EI): calcd. for $\mathrm{C}_{18} \mathrm{H}_{17} \mathrm{NO}_{2}[\mathrm{M}]^{+}$279.1259; found 279.1223 .

$\mathrm{N}$-Benzyl- $\boldsymbol{N}$-(2-hydroxyethyl)pent-2-ynamide (3d): Elution with heptane/EtOAc $(80 \%)$ delivered pure 3d as a ca. 3:7 mixture of rotamers, yield $180 \mathrm{mg}, 39 \%$. ${ }^{1} \mathrm{H}$ NMR $\left(300 \mathrm{MHz}, \mathrm{CDCl}_{3}\right): \delta=$ 7.45-7.15 (m, $5 \mathrm{H}), 4.87$ (s, $1.4 \mathrm{H}), 4.72(\mathrm{~s}, 0.6 \mathrm{H}), 3.82-3.74(\mathrm{~m}$, $0.6 \mathrm{H}), 3.73-3.59$ (m, $2 \mathrm{H}), 3.54-3.42(\mathrm{~m}, 1.4 \mathrm{H}), 3.05$ (br. s, $1 \mathrm{H})$,

$1612.44-2.29(\mathrm{~m}, 2 \mathrm{H}), 1.25-1.12(\mathrm{~m}, 3 \mathrm{H}) \mathrm{ppm} .{ }^{13} \mathrm{C}$ NMR $(75 \mathrm{MHz}$, $\left.\mathrm{CDCl}_{3}\right): \delta=156.4,155.5,136.8,136.3,128.8,128.7,128.1,128.0$, 127.6, 95.6, 95.1, 73.4, 73.3, 61.0, 60.6, 54.1, 50.3, 48.4, 47.5, 12.83, 12.75, 12.69 ppm. HRMS (EI): calcd. for $\mathrm{C}_{14} \mathrm{H}_{17} \mathrm{NO}_{2}[\mathrm{M}]^{+}$ 231.1259; found 231.1262

$166 \mathrm{~N}$-Benzyl- $\mathrm{N}$-(2-hydroxyethyl)oct-2-ynamide (3e): Elution with heptane/EtOAc $(50 \rightarrow 80 \%)$ delivered pure $3 \mathbf{e}$ as a ca. $3: 7$ mixture of rotamers, yield $262 \mathrm{mg}, 48 \% .{ }^{1} \mathrm{H}$ NMR $\left(300 \mathrm{MHz}, \mathrm{CDCl}_{3}\right): \delta=$ 7.41-7.18 (m, $5 \mathrm{H}), 4.88(\mathrm{~s}, 1.4 \mathrm{H}), 4.72(\mathrm{~s}, 0.6 \mathrm{H}), 3.82-3.74(\mathrm{~m}$, $0.6 \mathrm{H}), 3.73-3.58(\mathrm{~m}, 2 \mathrm{H}), 3.53-3.42(\mathrm{~m}, 1.4 \mathrm{H}), 2.39-2.27$ (m, 2

171 H), 1.65-1.44 (m, $2 \mathrm{H}), 1.41-1.19(\mathrm{~m}, 4 \mathrm{H}), 0.95-0.76(\mathrm{~m}, 3 \mathrm{H})$ ppm. ${ }^{13} \mathrm{C}$ NMR $\left(75 \mathrm{MHz}, \mathrm{CDCl}_{3}\right): \delta=156.4,155.5,136.8,136.3$, $128.8,128.7,128.1,127.9,127.52,127.47,94.6,94.1,74.0,73.9$, $60.9,60.7,54.1,50.3,48.4,47.5,31.04,30.95,27.5,27.3,22.1,22.0$, $18.91,18.89,13.9,13.8$ ppm. HRMS (EI): calcd. for $\mathrm{C}_{17} \mathrm{H}_{23} \mathrm{NO}_{2}$

\section{$176[\mathrm{M}]^{+}$273.1729; found 273.1738.}

General Procedure for the Synthesis of Hydroxypropargylamides 6 through the Ugi Reaction: 3-Substituted propiolic acid $(1.5 \mathrm{mmol})$ was placed in a screw-cap vial and dissolved in methanol $(2 \mathrm{~mL})$, and aldehyde $(1.5 \mathrm{mmol})$, ethanolamine $(92 \mathrm{mg}, 1.5 \mathrm{mmol})$, and isocyanide $(1.5 \mathrm{mmol})$ were added. The mixture was stirred at room temperature for $24 \mathrm{~h}$. Upon completion of the reaction, the resulting mixture was diluted with DCM and evaporated, and silica gel column chromatography delivered pure 6 .

$\mathrm{N}$-tert-Butyl-2-[N-(2-hydroxyethyl)but-2-ynamido|pentanamide (6a): 186 Elution with heptane/EtOAc/MeOH (50:50:1) delivered pure 6a as ca. $3: 7$ mixture of rotamers, yield $241 \mathrm{mg}, 57 \%$. ${ }^{1} \mathrm{H}$ NMR $\left(300 \mathrm{MHz}, \mathrm{CDCl}_{3}\right): \delta=6.51-6.21(\mathrm{~m}, 1 \mathrm{H}), 4.62-4.46(\mathrm{~m}, 0.3 \mathrm{H})$, $4.38(\mathrm{t}, J=7.8 \mathrm{~Hz}, 0.7 \mathrm{H}), 4.05-3.48(\mathrm{~m}, 4 \mathrm{H}), 2.03(\mathrm{~s}, 0.9 \mathrm{H}), 2.02$ $(\mathrm{s}, 2.1 \mathrm{H}), 1.99-1.70(\mathrm{~m}, 2 \mathrm{H}), 1.43-1.21(\mathrm{~m}, 11 \mathrm{H}), 1.02-0.90(\mathrm{~m}$,

$1913 \mathrm{H}) \mathrm{ppm} .{ }^{13} \mathrm{C}$ NMR $\left(75 \mathrm{MHz}, \mathrm{CDCl}_{3}\right): \delta=171.5,169.7,156.7$, $156.0,91.4,90.7,73.4,73.3,63.3,62.5,61.2,59.1,51.7,51.6,50.1$, $31.3,30.2,28.5,28.4,19.5,13.84,13.78,4.15,4.12 \mathrm{ppm}$. HRMS (ESI): calcd. for $\mathrm{C}_{15} \mathrm{H}_{27} \mathrm{~N}_{2} \mathrm{O}_{3}[\mathrm{M}+\mathrm{H}]^{+}$283.2016; found 283.2020.

$N$-[1-(tert-Butylamino)-1-oxopentan-2-yl]- $N$-(2-hydroxyethyl)pent-2ynamide (6b): Elution with heptane/EtOAc/MeOH (50:50:1) delivered pure $6 \mathbf{b}$ as a ca. 1:3 mixture of rotamers, yield $187 \mathrm{mg}, 42 \%$. ${ }^{1} \mathrm{H}$ NMR $\left(300 \mathrm{MHz}, \mathrm{CDCl}_{3}\right.$ ): $\delta=6.56$ (br. s, $0.25 \mathrm{H}$ ), 6.42 (br. s, $0.75 \mathrm{H}), 4.60-4.47(\mathrm{~m}, 0.25 \mathrm{H}), 4.41(\mathrm{t}, J=7.8 \mathrm{~Hz}, 0.75 \mathrm{H}), 4.01-$ $3.47(\mathrm{~m}, 4 \mathrm{H}), 2.47-2.31(\mathrm{~m}, 2 \mathrm{H}), 1.99-1.57$ (m, 2 H), 1.44-1.14 $(\mathrm{m}, 14 \mathrm{H}), 1.02-0.88(\mathrm{~m}, 3 \mathrm{H}) \mathrm{ppm} .{ }^{13} \mathrm{C} \mathrm{NMR}\left(75 \mathrm{MHz}, \mathrm{CDCl}_{3}\right.$, major rotamer): $\delta=171.5,156.1,95.8,73.4,62.3,59.1,51.6,49.9$, $30.3,28.4,19.5,13.8,12.70,12.65$ ppm. HRMS (ESI): calcd. for $\mathrm{C}_{16} \mathrm{H}_{29} \mathrm{~N}_{2} \mathrm{O}_{3}[\mathrm{M}+\mathrm{H}]^{+}$297.2173; found 297.2175.

$\mathrm{N}$-[1-(Cyclohexylamino)-1-oxopentan-2-yl]- $\mathrm{N}$-(2-hydroxyethyl)pent2-ynamide (6c): Elution with heptane/EtOAc/MeOH (50:50:1) delivered pure $\mathbf{6 c}$ as a ca. 1:3 mixture of rotamers, yield $290 \mathrm{mg}, 60 \%$. ${ }^{1} \mathrm{H}$ NMR $\left(300 \mathrm{MHz}, \mathrm{CDCl}_{3}\right): \delta=6.81(\mathrm{~d}, J=6.7 \mathrm{~Hz}, 0.25 \mathrm{H})$, $6.56(\mathrm{~d}, J=8.1 \mathrm{~Hz}, 0.75 \mathrm{H}), 5.12$ (br. s, $0.75 \mathrm{H}), 4.73-4.59$ (m, 0.25 $\mathrm{H}), 4.45(\mathrm{t}, J=7.8 \mathrm{~Hz}, 0.75 \mathrm{H}), 4.04-3.39(\mathrm{~m}, 5.25 \mathrm{H}), 2.46-2.30$ $(\mathrm{m}, 2 \mathrm{H}), 2.20-1.50(\mathrm{~m}, 7 \mathrm{H}), 1.45-1.05(\mathrm{~m}, 10 \mathrm{H}), 1.02-0.89(\mathrm{~m}$, $3 \mathrm{H}) \mathrm{ppm} .{ }^{13} \mathrm{C}$ NMR $\left(75 \mathrm{MHz}, \mathrm{CDCl}_{3}\right.$, major rotamer): $\delta=171.3$, 156.0, 95.8, 73.4, 62.3, 58.8, 50.2, 48.6, 32.6, 32.5, 30.4, 25.4, 24.7, 19.5, 13.8, 12.71, 12.65 ppm. HRMS (EI): calcd. for $\mathrm{C}_{18} \mathrm{H}_{30} \mathrm{~N}_{2} \mathrm{O}_{3}$ $[\mathrm{M}]^{+}$322.2256; found 322.2276 .

$\mathrm{N}$-Benzyl-2-[ $\mathrm{N}$-(2-hydroxyethyl)but-2-ynamido|pentanamide (6d): Elution with heptane/EtOAc/MeOH (60:40:1) delivered pure 6d as a ca. 1:2 mixture of rotamers, yield $237 \mathrm{mg}, 50 \%$. ${ }^{1} \mathrm{H}$ NMR $\left(300 \mathrm{MHz}, \mathrm{CDCl}_{3}\right): \delta=7.48-7.11(\mathrm{~m}, 5.33 \mathrm{H}), 6.94(\mathrm{t}, J=5.5 \mathrm{~Hz}$, $0.67 \mathrm{H}), 4.86-4.58(\mathrm{~m}, 1 \mathrm{H}), 4.54-4.31(\mathrm{~m}, 2.67 \mathrm{H}), 3.99-3.35(\mathrm{~m}$, $4.33 \mathrm{H}), 2.20-2.03(\mathrm{~m}, 0.33 \mathrm{H}), 2.00(\mathrm{~s}, 2 \mathrm{H}), 1.97-1.68(\mathrm{~m}, 2.67$ $\mathrm{H}), 1.45-1.20(\mathrm{~m}, 2 \mathrm{H}), 1.03-0.88(\mathrm{~m}, 3 \mathrm{H}) \mathrm{ppm} .{ }^{13} \mathrm{C}$ NMR $\left(75 \mathrm{MHz}, \mathrm{CDCl}_{3}\right): \delta=172.2,170.6,156.9,156.1,138.0,137.7$, $128.68,128.65,127.8,127.6,127.5,91.7,90.7,73.3,73.2,63.0,62.0$, $60.8,58.9,50.4,43.74,43.69,31.4,30.3,19.6,19.5,13.8,13.7,4.1$, 4.0 ppm. HRMS (EI): calcd. for $\mathrm{C}_{18} \mathrm{H}_{24} \mathrm{~N}_{2} \mathrm{O}_{3}[\mathrm{M}]^{+} 316.1787$; found 316.1756 .

2-[N-(2-Hydroxyethyl)but-2-ynamido]- $N$-(2,4,4-trimethylpentan-2yl)pentanamide (6e): Elution with heptane/EtOAc/MeOH (60:40:1) delivered pure 6e as a ca. 3:7 mixture of rotamers, yield $239 \mathrm{mg}$, $47 \%$. ${ }^{1} \mathrm{H}$ NMR $\left(300 \mathrm{MHz}, \mathrm{CDCl}_{3}\right): \delta=6.55-6.15(\mathrm{~m}, 1 \mathrm{H}), 4.69$ $4.49(\mathrm{~m}, 0.3 \mathrm{H}), 4.40(\mathrm{t}, J=7.6 \mathrm{~Hz}, 0.7 \mathrm{H}), 4.05-3.40(\mathrm{~m}, 4 \mathrm{H})$, $2.02(\mathrm{~s}, 3 \mathrm{H}), 1.97-1.55(\mathrm{~m}, 4 \mathrm{H}), 1.46-1.21(\mathrm{~m}, 8 \mathrm{H}), 1.05-0.90(\mathrm{~m}$, $12 \mathrm{H}$ ) ppm. ${ }^{13} \mathrm{C} \mathrm{NMR}\left(75 \mathrm{MHz}, \mathrm{CDCl}_{3}\right.$, major rotamer): $\delta=170.9$, 156.0, 90.7, 73.3, 62.4, 59.3, 55.7, 52.0, 50.2, 31.6, 31.4, 30.2, 28.7, $28.4,19.5,13.8,4.1 \mathrm{ppm}$. HRMS (EI): calcd. for $\mathrm{C}_{19} \mathrm{H}_{34} \mathrm{~N}_{2} \mathrm{O}_{3}$ $[\mathrm{M}]^{+}$338.2569; found 338.2597.

$\mathrm{N}$-tert-Butyl-2-[N-(2-hydroxyethyl)-3-phenylpropiolamido|pentanamide (6f): Elution with heptane/EtOAc/MeOH (70:30:1) delivered pure 6 f as a ca. 1:3 mixture of rotamers, yield $233 \mathrm{mg}, 45 \% .{ }^{1} \mathrm{H}$ NMR $\left(300 \mathrm{MHz}, \mathrm{CDCl}_{3}\right): \delta=7.64-7.49(\mathrm{~m}, 2 \mathrm{H}), 7.48-7.30(\mathrm{~m}, 3$ H), 6.68 (br. s, $0.25 \mathrm{H}$ ), 6.37 (br. s, $0.75 \mathrm{H}$ ), 4.99 (br. s, $0.75 \mathrm{H}$ ), 4.65-4.30 (m, 1 H), 4.12-3.43 (m, 4.25 H), 2.24-2.05 (m, 0.25 H), $2.03-1.74(\mathrm{~m}, 1.75 \mathrm{H}), 1.47-1.21(\mathrm{~m}, 11 \mathrm{H}), 0.98(\mathrm{t}, J=7.3 \mathrm{~Hz}, 3$ H) ppm. ${ }^{13} \mathrm{C}$ NMR $\left(75 \mathrm{MHz}, \mathrm{CDCl}_{3}\right): \delta=171.4,169.7,156.5$, $156.0,132.6,132.5,130.5,130.3,128.64,128.59,120.1,120.0,92.0$, $91.6,81.64,81.56,63.7,62.5,60.9,59.4,51.8,51.6,50.3,31.7,30.4$, $28.5,19.8,19.6,13.88,13.85 \mathrm{ppm}$. HRMS (EI): calcd. for $\mathrm{C}_{20} \mathrm{H}_{28} \mathrm{~N}_{2} \mathrm{O}_{3}[\mathrm{M}]^{+}$344.2100; found 344.2147.

$\mathrm{N}$-[1-(tert-Butylamino)-1-oxo-3-phenylpropan-2-yl]- $N$-(2-hydroxyethyl)but-2-ynamide (6g): Elution with heptane/EtOAc/MeOH (60:40:1) delivered pure $\mathbf{6 g}$ as a ca. 1:3 mixture of rotamers, yield $248 \mathrm{mg}, 50 \%$. ${ }^{1} \mathrm{H}$ NMR $\left(300 \mathrm{MHz}, \mathrm{CDCl}_{3}\right): \delta=7.36-7.11(\mathrm{~m}, 5$ H), 6.70 (br. s, $0.25 \mathrm{H}$ ), 6.04 (br. s, $0.75 \mathrm{H}$ ), 4.91 (br. s, $0.75 \mathrm{H}$ ), $4.47(\mathrm{t}, J=8.0 \mathrm{~Hz}, 1 \mathrm{H}), 3.84-3.36(\mathrm{~m}, 4.25 \mathrm{H}), 3.31-3.11(\mathrm{~m}, 1.75$ H), 2.83 (br. s, $0.25 \mathrm{H}), 2.01(\mathrm{~s}, 0.75 \mathrm{H}), 1.99(\mathrm{~s}, 2.25 \mathrm{H}), 1.32(\mathrm{~s}$, $2.5 \mathrm{H}), 1.25(\mathrm{~s}, 7.5 \mathrm{H}) \mathrm{ppm} .{ }^{13} \mathrm{C}$ NMR $\left(75 \mathrm{MHz}, \mathrm{CDCl}_{3}\right): \delta=170.5$ $168.9,156.1,155.8,137.6,136.7,129.2,129.1,128.7,126.9,126.8$, $91.4,90.5,73.7,73.4,65.6,62.0,61.6,60.5,51.7,51.5,51.2,35.9$ 34.3, 28.4, 28.3, 4.2, 4.1 ppm. HRMS (ESI): calcd. for $\mathrm{C}_{19} \mathrm{H}_{27} \mathrm{~N}_{2} \mathrm{O}_{3}$ $[\mathrm{M}+\mathrm{H}]^{+}$331.2016; found 331.2019.
201 
$\mathrm{N}$-tert-Butyl-2-[ $\mathrm{N}$-(2-hydroxyethyl)but-2-ynamido]-4-methylpentanamide (6h): Elution with heptane/EtOAc/MeOH (50:50:1) delivered pure $6 \mathbf{h}$ as a ca. $3: 7$ mixture of rotamers, yield $369 \mathrm{mg}, 83 \% .{ }^{1} \mathrm{H}$ NMR $\left(300 \mathrm{MHz}, \mathrm{CDCl}_{3}\right): \delta=6.57-6.26(\mathrm{~m}, 1 \mathrm{H}), 4.85-4.44(\mathrm{~m}$, $2661.7 \mathrm{H}), 4.08-3.40(\mathrm{~m}, 4.3 \mathrm{H}), 2.04(\mathrm{~s}, 0.9 \mathrm{H}), 2.02(\mathrm{~s}, 2.1 \mathrm{H}), 1.97-$ $1.43(\mathrm{~m}, 3 \mathrm{H}), 1.35(\mathrm{~s}, 2.7 \mathrm{H}), 1.33(\mathrm{~s}, 6.3 \mathrm{H}), 1.01-0.89(\mathrm{~m}, 6 \mathrm{H})$ ppm. ${ }^{13} \mathrm{C}$ NMR $\left(75 \mathrm{MHz}, \mathrm{CDCl}_{3}\right): \delta=171.5,169.7,156.6,156.1$, $91.3,90.7,73.7,73.4,73.3,62.3,61.5,61.0,56.9,51.60,51.57,49.8$, $38.1,36.8,35.4,28.5,28.4,24.8,24.6,23.1,22.8,22.3,21.8,4.10$,

2714.09 ppm. HRMS (EI): calcd. for $\mathrm{C}_{16} \mathrm{H}_{28} \mathrm{~N}_{2} \mathrm{O}_{3}[\mathrm{M}]^{+}$296.2100; found 296.2111 .

General Procedure for the Synthesis of Hydroxypropargylamides 7 through the Ugi Reaction: Tetrolic acid $(126 \mathrm{mg}, 1.5 \mathrm{mmol})$ was placed in a screw-cap vial and dissolved in methanol $(2 \mathrm{~mL})$, and glycolaldehyde dimer $(90 \mathrm{mg}, 0.75 \mathrm{mmol})$, the appropriate amine (92 $\mathrm{mg}, 1.5 \mathrm{mmol})$, and tert-butyl isocyanide $(125 \mathrm{mg}, 1.5 \mathrm{mmol})$ were added. The mixture was stirred at room temperature for $24 \mathrm{~h}$. Upon completion of the reaction, the resulting mixture was diluted with DCM and evaporated, and silica gel column chromatography 281 delivered pure 7.

$N$-Butyl- $N$-[1-(tert-butylamino)-3-hydroxy-1-oxopropan-2-yl]but-2ynamide (7a): Elution with heptane/EtOAc $(50 \rightarrow 80 \%)$ delivered pure $7 \mathbf{a}$ as a ca. $1: 4$ mixture of rotamers, yield $288 \mathrm{mg}, 68 \%{ }^{1} \mathrm{H}$ NMR ( $300 \mathrm{MHz}, \mathrm{CDCl}_{3}$ ): $\delta=6.66$ (br. s, $0.8 \mathrm{H}$ ), 6.11 (br. s, 0.2

$286 \mathrm{H}), 4.81-4.72(\mathrm{~m}, 0.2 \mathrm{H}), 4.65(\mathrm{t}, J=5.9 \mathrm{~Hz}, 0.8 \mathrm{H}), 4.22-4.10(\mathrm{~m}$, $0.2 \mathrm{H}), 4.09-3.96$ (m, 0.8), 3.96-3.53 (m, 3.6 H), 3.52-3.39 (m, 0.2 H), 3.17-3.03 (m, $0.2 \mathrm{H}), 2.04(\mathrm{~s}, 2.4 \mathrm{H}), 2.02(\mathrm{~s}, 0.6 \mathrm{H}), 1.75-1.43$ $(\mathrm{m}, 2 \mathrm{H}), 1.42-1.22(\mathrm{~m}, 11 \mathrm{H}), 1.00-0.83(\mathrm{~m}, 3 \mathrm{H}) \mathrm{ppm} .{ }^{13} \mathrm{C} \mathrm{NMR}$ $\left(75 \mathrm{MHz}, \mathrm{CDCl}_{3}\right): \delta=169.5,169.2,156.2,156.0,90.5,90.3,73.2$,

$29163.3,61.7,60.8,58.7,51.8,51.3,48.1,44.8,31.7,30.4,28.52,28.49$, $20.4,20.0,13.63,13.55,4.1,4.0 \mathrm{ppm}$. HRMS (ESI): calcd. for $\mathrm{C}_{15} \mathrm{H}_{27} \mathrm{~N}_{2} \mathrm{O}_{3}[\mathrm{M}+\mathrm{H}]^{+}$283.2016; found 283.2016.

$\mathrm{N}$-[1-(tert-Butylamino)-3-hydroxy-1-oxopropan-2-yl]- $N$-(4-methoxybenzyl)but-2-ynamide (7b): Elution with heptane/EtOAc

$296(50 \rightarrow 80 \%)$ delivered pure $7 \mathbf{b}$ as a ca. 1:2 mixture of rotamers, yield $208 \mathrm{mg}, 40 \%$. ${ }^{1} \mathrm{H}$ NMR $\left(300 \mathrm{MHz}, \mathrm{CDCl}_{3}\right): \delta=7.37-7.23(\mathrm{~m}, 2$ H), 6.96-6.80 (m, $2 \mathrm{H}), 6.07$ (br. s, $0.67 \mathrm{H}), 5.34$ (br. s, $0.33 \mathrm{H}$ ), 5.13 (br. d, $J=14.4 \mathrm{~Hz}, 0.33 \mathrm{H}), 4.98(\mathrm{~d}, J=15.6 \mathrm{~Hz}, 0.67 \mathrm{H})$, $4.72(\mathrm{~d}, J=15.6 \mathrm{~Hz}, 0.67 \mathrm{H}), 4.53-4.19(\mathrm{~m}, 1.33 \mathrm{H}), 4.10-3.91(\mathrm{~m}$,

$3011 \mathrm{H}), 3.80$ (s, $2 \mathrm{H}), 3.79(\mathrm{~s}, 1 \mathrm{H}), 3.75-3.61(\mathrm{~m}, 1 \mathrm{H}), 3.52$ (br. s, $0.67 \mathrm{H}), 3.36$ (br. s, $0.33 \mathrm{H}), 2.04$ (s, $2 \mathrm{H}), 1.99$ (s, $1 \mathrm{H}), 1.19$ (s, 6 $\mathrm{H}), 1.15(\mathrm{~s}, 3 \mathrm{H}) \mathrm{ppm} .{ }^{13} \mathrm{C}$ NMR $\left(75 \mathrm{MHz}, \mathrm{CDCl}_{3}\right): \delta=169.0$, $168.7,159.5,155.9,130.0,129.4,129.1,128.7,114.5,114.4,91.4$, $91.2,73.4,73.3,62.9,61.7,60.9,59.6,55.3,52.2,51.5,51.3,35.4$,

306 28.5, 28.2, 4.12, 4.09 ppm. HRMS (ESI): calcd. for $\mathrm{C}_{19} \mathrm{H}_{27} \mathrm{~N}_{2} \mathrm{O}_{4}$ $[\mathrm{M}+\mathrm{H}]^{+}$347.1965; found 347.1960.

General Procedure for the Gold-Catalyzed Cycloisomerization of Hydroxypropargylamides 3, 6, and 7 into Oxazepines 4 (Conditions A): Hydroxypropargylamide $\mathbf{3}, \mathbf{6}$, or $\mathbf{7}(0.3 \mathrm{mmol})$ was dissolved in dry $\mathrm{CHCl}_{3}(1.2 \mathrm{~mL})$, and $\mathrm{AuPPh}_{3} \mathrm{Cl}(7.4 \mathrm{mg}, 0.015 \mathrm{mmol})$ and $\mathrm{Ag}$ OTf $(3.9 \mathrm{mg}, 0.015 \mathrm{mmol})$ were added. The resulting mixture was stirred at room temp. for $2-4 \mathrm{~h}$ in a sealed screw-cap vial. The resulting mixture was concentrated under reduced pressure and loaded onto a silica gel column to deliver pure 4 .

316 General Procedure for the Silver-Catalyzed Cycloisomerization of Hydroxypropargylamides 3, 6, and 7 into Oxazepines 4 (Conditions B): Hydroxypropargylamide $\mathbf{3}, \mathbf{6}$, or $\mathbf{7}(0.3 \mathrm{mmol})$ was dissolved in dry $\mathrm{CHCl}_{3}(1.2 \mathrm{~mL})$, and $\mathrm{AgOTf}(3.9 \mathrm{mg}, 0.015 \mathrm{mmol})$ was added. The resulting mixture was stirred at $50^{\circ} \mathrm{C}$ for $15 \mathrm{~h}$ in a sealed

321 screw-cap vial. The resulting mixture was concentrated under reduced pressure and loaded onto a silica gel column to deliver pure 4.
4-Benzyl-7-methyl-3,4-dihydro-1,4-oxazepin-5(2H)-one (4a): Elution with heptane/EtOAc $(50 \rightarrow 70 \%)$ provided pure $4 \mathrm{a}$, yield $62.6 \mathrm{mg}$, $96 \%$ (procedure A); $59.3 \mathrm{mg}, 91 \%$ (procedure B). ${ }^{1} \mathrm{H}$ NMR $\left(300 \mathrm{MHz}, \mathrm{CDCl}_{3}\right): \delta=7.40-7.12(\mathrm{~m}, 5 \mathrm{H}), 5.21(\mathrm{~s}, 1 \mathrm{H}), 4.63(\mathrm{~s}$, $2 \mathrm{H}), 4.22-4.03(\mathrm{~m}, 2 \mathrm{H}), 3.52-3.33(\mathrm{~m}, 2 \mathrm{H}), 1.93$ (s, $3 \mathrm{H}) \mathrm{ppm}$. ${ }^{13} \mathrm{C}$ NMR $\left(75 \mathrm{MHz}, \mathrm{CDCl}_{3}\right): \delta=166.6,160.3,137.0,128.7,128.0$, 127.5, 99.1, 71.4, 51.4, 47.9, 22.6 ppm. HRMS (EI): calcd. for $\mathrm{C}_{13} \mathrm{H}_{15} \mathrm{NO}_{2}[\mathrm{M}]^{+}$217.1103; found 217.1122.

(S)-3-Methyl-7,8,9,9a-tetrahydropyrrolo[2,1-c][1,4]oxazepin-5(1H)one $[(S)-\mathbf{4 b}]$ : Elution with pure EtOAc provided pure $(S)-\mathbf{4 b}$, yield $47.7 \mathrm{mg}, 95 \%$ (procedure A); $44.6 \mathrm{mg}, 89 \%$ (procedure B). $[\alpha]_{\mathrm{D}}^{20}=$ $-80.1\left(c=1, \mathrm{CHCl}_{3}\right) .{ }^{1} \mathrm{H}$ NMR $\left(300 \mathrm{MHz}, \mathrm{CDCl}_{3}\right): \delta=5.10(\mathrm{~s}, 1$ H), $4.39(\mathrm{~d}, J=11.7 \mathrm{~Hz}, 1 \mathrm{H}), 3.92-3.63(\mathrm{~m}, 3 \mathrm{H}), 3.53-3.39(\mathrm{~m}$, $2 \mathrm{H}), 2.26-2.12(\mathrm{~m}, 2 \mathrm{H}), 2.07-1.73(\mathrm{~m}, 5 \mathrm{H}), 1.67-1.49(\mathrm{~m}, 1 \mathrm{H})$ ppm. ${ }^{13} \mathrm{C}$ NMR $\left(75 \mathrm{MHz}, \mathrm{CDCl}_{3}\right): \delta=164.3,161.1,99.0,74.2$, 57.6, 47.3, 29.7, 22.8, 22.7 ppm. HRMS (EI): calcd. for $\mathrm{C}_{13} \mathrm{H}_{15} \mathrm{NO}_{2}$ $[\mathrm{M}]^{+}$167.0946; found 167.0942.

(R)-3-Methyl-7,8,9,9a-tetrahydropyrrolo[2,1-c|[1,4]oxazepin-5(1H)one $[(\boldsymbol{R})-\mathbf{4 b}]$ : Elution with pure EtOAc provided pure $(R)-\mathbf{4 b}$, yield $48.2 \mathrm{mg}, 96 \%$ (procedure A). $[\alpha]_{\mathrm{D}}^{20}=+81.8\left(c=0.6, \mathrm{CHCl}_{3}\right)$.

4-Benzyl-7-phenyl-3,4-dihydro-1,4-oxazepin-5(2H)-one (4c): Elution with heptane/EtOAc (30\%) provided pure 4c, yield $61.2 \mathrm{mg}, 73 \%$ (procedure A); $62.0 \mathrm{mg}, 74 \%$ (procedure B). ${ }^{1} \mathrm{H}$ NMR $(300 \mathrm{MHz}$, $\left.\mathrm{CDCl}_{3}\right): \delta=7.71-7.60(\mathrm{~m}, 2 \mathrm{H}), 7.45-7.21(\mathrm{~m}, 8 \mathrm{H}), 5.93(\mathrm{~s}, 1 \mathrm{H})$, $4.72(\mathrm{~s}, 2 \mathrm{H}), 4.45-4.33(\mathrm{~m}, 2 \mathrm{H}), 3.63-3.52(\mathrm{~m}, 2 \mathrm{H}) \mathrm{ppm} .{ }^{13} \mathrm{C}$ NMR $\left(75 \mathrm{MHz}, \mathrm{CDCl}_{3}\right): \delta=166.7,159.1,136.9,135.5,130.1$, 128.8, 128.4, 128.1, 127.6, 126.6, 98.8, 71.7, 51.7, 48.2 ppm. HRMS (EI): calcd. for $\mathrm{C}_{18} \mathrm{H}_{17} \mathrm{NO}_{2}[\mathrm{M}]^{+}$279.1259; found 279.1262 .

4-Benzyl-7-ethyl-3,4-dihydro-1,4-oxazepin-5(2H)-one (4d): Elution with heptane/EtOAc $(40 \%)$ provided pure 4 d, yield $58.3 \mathrm{mg}, 84 \%$ (procedure A); $59.7 \mathrm{mg}, 86 \%$ (procedure B). ${ }^{1} \mathrm{H}$ NMR $(300 \mathrm{MHz}$, $\left.\mathrm{CDCl}_{3}\right): \delta=7.40-7.18(\mathrm{~m}, 5 \mathrm{H}), 5.22(\mathrm{~s}, 1 \mathrm{H}), 4.65(\mathrm{~s}, 2 \mathrm{H}), 4.22$ $4.10(\mathrm{~m}, 2 \mathrm{H}), 3.50-3.38(\mathrm{~m}, 2 \mathrm{H}), 2.20$ (q, $J=7.5 \mathrm{~Hz}, 2 \mathrm{H}), 1.11$ $(\mathrm{t}, J=7.5 \mathrm{~Hz}, 3 \mathrm{H}) \mathrm{ppm} .{ }^{13} \mathrm{C} \mathrm{NMR}\left(75 \mathrm{MHz}, \mathrm{CDCl}_{3}\right): \delta=166.9$, $164.9,137.1,128.7,128.1,127.5,97.7,71.4,51.5,48.0,29.6,12.1$ ppm. HRMS (EI): calcd. for $\mathrm{C}_{14} \mathrm{H}_{17} \mathrm{NO}_{2}[\mathrm{M}]^{+}$231.1259; found 231.1244 .

4-Benzyl-7-pentyl-3,4-dihydro-1,4-oxazepin-5(2H)-one (4e): Elution with heptane/EtOAc (40\%) provided pure 4e, yield $73.8 \mathrm{mg}, 90 \%$ (procedure A); $70.5 \mathrm{mg}, 86 \%$ (procedure B). ${ }^{1} \mathrm{H}$ NMR $(300 \mathrm{MHz}$, $\left.\mathrm{CDCl}_{3}\right): \delta=7.38-7.18(\mathrm{~m}, 5 \mathrm{H}), 5.22(\mathrm{~s}, 1 \mathrm{H}), 4.65(\mathrm{~s}, 2 \mathrm{H}), 4.22-$ 4.08 (m, $2 \mathrm{H}), 3.49-3.37$ (m, $2 \mathrm{H}), 2.23-2.08$ (m, $2 \mathrm{H}), 1.61-1.45$ $(\mathrm{m}, 2 \mathrm{H}), 1.41-1.18(\mathrm{~m}, 4 \mathrm{H}), 0.89(\mathrm{t}, J=6.9 \mathrm{~Hz}, 3 \mathrm{H}) \mathrm{ppm} .{ }^{13} \mathrm{C}$ NMR $\left(75 \mathrm{MHz}, \mathrm{CDCl}_{3}\right): \delta=166.8,163.8,137.1,128.7,128.1$, 127.5, 98.6, 71.4, 51.4, 48.0, 36.5, 31.2, 27.3, 22.4, 14.0 ppm. HRMS (EI): calcd. for $\mathrm{C}_{17} \mathrm{H}_{23} \mathrm{NO}_{2}[\mathrm{M}]^{+}$273.1729; found 273.1691.

$\mathrm{N}$-tert-Butyl-2-[7-methyl-5-oxo-2,3-dihydro-1,4-oxazepin-4(5H)-yl]pentanamide (4f): Elution with heptane/EtOAc (50\%) provided pure 4f, yield $75.3 \mathrm{mg}, 89 \%$ (procedure A); $75.4 \mathrm{mg}, 89 \%$ (procedure B). ${ }^{1} \mathrm{H}$ NMR $\left(300 \mathrm{MHz}, \mathrm{CDCl}_{3}\right.$ ): $\delta=5.99$ (br. s, $1 \mathrm{H}$ ), 5.15 $(\mathrm{s}, 1 \mathrm{H}), 4.89(\mathrm{t}, J=7.7 \mathrm{~Hz}, 1 \mathrm{H}), 4.37(\mathrm{dd}, J=12.4,6.3 \mathrm{~Hz}, 1 \mathrm{H})$, $4.06(\mathrm{dd}, J=12.4,6.7 \mathrm{~Hz}, 1 \mathrm{H}), 3.68(\mathrm{dd}, J=15.9,6.3 \mathrm{~Hz}, 1 \mathrm{H})$, $3.47(\mathrm{dd}, J=15.9,6.7 \mathrm{~Hz}, 1 \mathrm{H}), 1.95(\mathrm{~s}, 3 \mathrm{H}), 1.91-1.71(\mathrm{~m}, 1 \mathrm{H})$, $1.65-1.45(\mathrm{~m}, 1 \mathrm{H}), 1.39-1.17(\mathrm{~m}, 11 \mathrm{H}), 0.92(\mathrm{t}, J=7.3 \mathrm{~Hz}, 3 \mathrm{H})$ ppm. ${ }^{13} \mathrm{C}$ NMR $\left(75 \mathrm{MHz}, \mathrm{CDCl}_{3}\right): \delta=169.8,167.3,160.7,98.6$, 72.0, 56.7, 51.2, 43.8, 30.2, 28.7, 22.6, 19.2, 13.9 ppm. HRMS (ESI): calcd. for $\mathrm{C}_{15} \mathrm{H}_{27} \mathrm{~N}_{2} \mathrm{O}_{3}[\mathrm{M}+\mathrm{H}]^{+}$283.2016; found 283.2020.

$\mathrm{N}$-tert-Butyl-2-[7-ethyl-5-oxo-2,3-dihydro-1,4-oxazepin-4(5H)-yl]pentanamide (4g): Elution with heptane/EtOAc $(40 \%)$ provided pure $4 \mathrm{~g}$, yield $66.7 \mathrm{mg}, 75 \%$ (procedure A); $56.9 \mathrm{mg}, 64 \%$ (procedure B). ${ }^{1} \mathrm{H}$ NMR $\left(300 \mathrm{MHz}, \mathrm{CDCl}_{3}\right.$ ): $\delta=5.99$ (br. s, $1 \mathrm{H}$ ), 5.14 
$(\mathrm{s}, 1 \mathrm{H}), 4.90(\mathrm{t}, J=7.7 \mathrm{~Hz}, 1 \mathrm{H}), 4.38(\mathrm{dd}, J=12.4,6.3 \mathrm{~Hz}, 1 \mathrm{H})$,

$(\mathrm{dd}, J=12.4,6.6 \mathrm{~Hz}, 1 \mathrm{H}), 3.68(\mathrm{dd}, J=15.9,6.3 \mathrm{~Hz}, 1 \mathrm{H})$ $3.46(\mathrm{dd}, J=15.9,6.6 \mathrm{~Hz}, 1 \mathrm{H}), 2.21(\mathrm{q}, J=7.5 \mathrm{~Hz}, 2 \mathrm{H}), 1.90$ $1.71(\mathrm{~m}, 1 \mathrm{H}), 1.65-1.47(\mathrm{~m}, 1 \mathrm{H}), 1.36-1.19(\mathrm{~m}, 11 \mathrm{H}), 1.12(\mathrm{t}, J$ $=7.5 \mathrm{~Hz}, 3 \mathrm{H}), 0.93(\mathrm{t}, J=7.3 \mathrm{~Hz}, 3 \mathrm{H}) \mathrm{ppm} .{ }^{13} \mathrm{C} \mathrm{NMR}(75 \mathrm{MHz}$, $\left.\mathrm{CDCl}_{3}\right): \delta=169.8,167.6,165.3,97.1,71.9,56.7,51.2,43.9,30.2$, 29.6, 28.7, 19.2, 13.9, $12.0 \mathrm{ppm}$. HRMS (ESI): calcd. for $\mathrm{C}_{16} \mathrm{H}_{29} \mathrm{~N}_{2} \mathrm{O}_{3}[\mathrm{M}+\mathrm{H}]^{+}$297.2173; found 297.2175.

$\mathrm{N}$-Cyclohexyl-2-[7-ethyl-5-oxo-2,3-dihydro-1,4-oxazepin-4(5H)-yl]pentanamide (4h): Elution with heptane/EtOAc (40\%) provided pure $4 \mathrm{~h}$, yield $74.5 \mathrm{mg}, 77 \%$ (procedure A); $71.6 \mathrm{mg}, 74 \%$ (procedure B). ${ }^{1} \mathrm{H}$ NMR $\left(300 \mathrm{MHz}, \mathrm{CDCl}_{3}\right.$ ): $\delta=6.12$ (br. d, $J=8.1 \mathrm{~Hz}$, $1 \mathrm{H}), 5.14(\mathrm{~s}, 1 \mathrm{H}), 4.98(\mathrm{dd}, J=8.3,7.2 \mathrm{~Hz}, 1 \mathrm{H}), 4.38(\mathrm{dd}, J=$ $12.4,6.3 \mathrm{~Hz}, 1 \mathrm{H}), 4.07(\mathrm{dd}, J=12.4,6.6 \mathrm{~Hz}, 1 \mathrm{H}), 3.78-3.58(\mathrm{~m}$, $2 \mathrm{H}), 3.47(\mathrm{dd}, J=15.9,6.6 \mathrm{~Hz}, 1 \mathrm{H}), 2.20(\mathrm{q}, J=7.5 \mathrm{~Hz}, 2 \mathrm{H})$, $1.93-1.51(\mathrm{~m}, 7 \mathrm{H}), 1.43-1.01(\mathrm{~m}, 10 \mathrm{H}), 0.93(\mathrm{t}, J=7.3 \mathrm{~Hz}, 3 \mathrm{H})$ ppm. ${ }^{13} \mathrm{C}$ NMR $\left(75 \mathrm{MHz}, \mathrm{CDCl}_{3}\right): \delta=169.6,167.7,165.4,97.1$, 71.9, 56.3, 48.1, 44.0, 33.0, 32.8, 30.4, 29.6, 25.5, 24.8, 24.7, 19.2, 13.9, 12.1 ppm. HRMS (EI): calcd. for $\mathrm{C}_{18} \mathrm{H}_{30} \mathrm{~N}_{2} \mathrm{O}_{3}[\mathrm{M}]^{+}$322.2256; found 322.2276 .

$\mathrm{N}$-Benzyl-2-[7-methyl-5-oxo-2,3-dihydro-1,4-oxazepin-4(5H)-yl]pentanamide (4i): Elution with heptane/EtOAc (40\%) provided pure $4 \mathbf{i}$, yield $83.5 \mathrm{mg}, 88 \%$ (procedure A); $77.8 \mathrm{mg}, 82 \%$ (procedure B). ${ }^{1} \mathrm{H}$ NMR $\left(300 \mathrm{MHz}, \mathrm{CDCl}_{3}\right): \delta=7.37-7.18(\mathrm{~m}, 5 \mathrm{H})$, $6.94(\mathrm{t}, J=5.6 \mathrm{~Hz}, 1 \mathrm{H}), 5.12-5.01(\mathrm{~m}, 2 \mathrm{H}), 4.37(\mathrm{~d}, J=6.0 \mathrm{~Hz}$, $2 \mathrm{H}), 4.26(\mathrm{dd}, J=12.4,6.3 \mathrm{~Hz}, 1 \mathrm{H}), 3.95(\mathrm{dd}, J=12.4,6.6 \mathrm{~Hz}$, $4111 \mathrm{H}), 3.65(\mathrm{dd}, J=15.9,6.3 \mathrm{~Hz}, 1 \mathrm{H}), 3.47(\mathrm{dd}, J=15.9,6.6 \mathrm{~Hz}$, $1 \mathrm{H}), 1.95-1.76(\mathrm{~m}, 4 \mathrm{H}), 1.71-1.52(\mathrm{~m}, 1 \mathrm{H}), 1.38-1.18(\mathrm{~m}, 2 \mathrm{H})$, $0.92(\mathrm{t}, J=7.3 \mathrm{~Hz}, 3 \mathrm{H}) \mathrm{ppm} .{ }^{13} \mathrm{C}$ NMR $\left(75 \mathrm{MHz}, \mathrm{CDCl}_{3}\right): \delta=$ 170.6, 167.4, 160.8, 138.3, 128.6, 127.7, 127.4, 98.4, 71.8, 56.2, 43.9, 43.3, 30.5, 22.6, 19.2, $13.9 \mathrm{ppm}$. HRMS (EI): calcd. for $\mathrm{C}_{18} \mathrm{H}_{24} \mathrm{~N}_{2} \mathrm{O}_{3}[\mathrm{M}]^{+}$316.1787; found 316.1788 .

2-[7-Methyl-5-oxo-2,3-dihydro-1,4-oxazepin-4(5H)-yl]- $N$-(2,4,4-trimethylpentan-2-yl)pentanamide (4j): Elution with heptane/EtOAc (30\%) provided pure $\mathbf{4 j}$, yield $88.3 \mathrm{mg}, 87 \%$ (procedure A); $85.3 \mathrm{mg}, 84 \%$ (procedure B). ${ }^{1} \mathrm{H}$ NMR $\left(300 \mathrm{MHz}, \mathrm{CDCl}_{3}\right): \delta=$ 6.01 (br. s, $1 \mathrm{H}), 5.14(\mathrm{~s}, 1 \mathrm{H}), 4.87(\mathrm{t}, J=7.7 \mathrm{~Hz}, 1 \mathrm{H}), 4.33(\mathrm{dd}$, $J=12.4,6.3 \mathrm{~Hz}, 1 \mathrm{H}), 4.12(\mathrm{dd}, J=12.4,6.5 \mathrm{~Hz}, 1 \mathrm{H}), 3.64(\mathrm{dd}$, $J=15.9,6.3 \mathrm{~Hz}, 1 \mathrm{H}), 3.49(\mathrm{dd}, J=15.9,6.5 \mathrm{~Hz}, 1 \mathrm{H}), 1.94(\mathrm{~s}, 3$ $\mathrm{H}), 1.89-1.70(\mathrm{~m}, 2 \mathrm{H}), 1.68-1.45(\mathrm{~m}, 2 \mathrm{H}), 1.41-1.14(\mathrm{~m}, 8 \mathrm{H})$, $0.98(\mathrm{~s}, 9 \mathrm{H}), 0.92(\mathrm{t}, J=7.3 \mathrm{~Hz}, 3 \mathrm{H}) \mathrm{ppm} .{ }^{13} \mathrm{C} \mathrm{NMR}(75 \mathrm{MHz}$, 源 31.6, 31.4, 30.2, 29.1, 29.0, 22.6, 19.3, 13.9 ppm. HRMS (EI): calcd. for $\mathrm{C}_{19} \mathrm{H}_{34} \mathrm{~N}_{2} \mathrm{O}_{3}[\mathrm{M}]^{+}$338.2569; found 338.2547.

$\mathrm{N}$-tert-Butyl-2-[5-oxo-7-phenyl-2,3-dihydro-1,4-oxazepin-4(5H)-yl]pentanamide (4k): Elution with heptane/EtOAc $(40 \%)$ provided pure $4 \mathbf{k}$, yield $92.0 \mathrm{mg}, 89 \%$ (procedure A); $80.6 \mathrm{mg}, 78 \%$ (procedure B). ${ }^{1} \mathrm{H}$ NMR $\left(300 \mathrm{MHz}, \mathrm{CDCl}_{3}\right): \delta=7.71-7.62(\mathrm{~m}, 2 \mathrm{H})$, 7.46-7.32 (m, $3 \mathrm{H}), 6.06$ (br. s, $1 \mathrm{H}), 5.84(\mathrm{~s}, 1 \mathrm{H}), 4.96(\mathrm{t}, J=$ $7.7 \mathrm{~Hz}, 1 \mathrm{H}), 4.62(\mathrm{dd}, J=12.4,6.3 \mathrm{~Hz}, 1 \mathrm{H}), 4.27(\mathrm{dd}, J=12.4$, $6.7 \mathrm{~Hz}, 1 \mathrm{H}), 3.82(\mathrm{dd}, J=15.9,6.3 \mathrm{~Hz}, 1 \mathrm{H}), 3.60(\mathrm{dd}, J=15.9$,

$436 \quad 6.7 \mathrm{~Hz}, 1 \mathrm{H}), 1.94-1.75(\mathrm{~m}, 1 \mathrm{H}), 1.70-1.51(\mathrm{~m}, 1 \mathrm{H}), 1.41-1.21(\mathrm{~m}$, $11 \mathrm{H}), 0.95$ (t, $J=7.3 \mathrm{~Hz}, 3 \mathrm{H}) \mathrm{ppm} .{ }^{13} \mathrm{C} \mathrm{NMR}\left(75 \mathrm{MHz}, \mathrm{CDCl}_{3}\right)$ : $\delta=169.7,167.3,159.3,135.4,130.1,128.4,126.5,98.2,72.3,56.9$, 51.3, 44.1, 30.3, 28.7, 19.3, $13.9 \mathrm{ppm}$. HRMS (EI): calcd. for $\mathrm{C}_{20} \mathrm{H}_{28} \mathrm{~N}_{2} \mathrm{O}_{3}[\mathrm{M}]^{+}$344.2100; found 344.2125.

$441 \quad \mathrm{~N}$-tert-Butyl-2-[7-methyl-5-oxo-2,3-dihydro-1,4-oxazepin-4(5H)-yl]3-phenylpropanamide (4I): Elution with heptane/EtOAc (50\%) provided pure $4 \mathbf{l}$, yield $83.3 \mathrm{mg}, 84 \%$ (procedure A); $85.2 \mathrm{mg}, 86 \%$ (procedure B). ${ }^{1} \mathrm{H}$ NMR $\left(300 \mathrm{MHz}, \mathrm{CDCl}_{3}\right): \delta=7.36-7.10(\mathrm{~m}, 5$ H), 5.95 (br. s, $1 \mathrm{H}), 5.25(\mathrm{t}, J=8.2 \mathrm{~Hz}, 1 \mathrm{H}), 5.08(\mathrm{~s}, 1 \mathrm{H}), 4.12$ $3.90(\mathrm{~m}, 2 \mathrm{H}), 3.77-3.62(\mathrm{~m}, 1 \mathrm{H}), 3.55-3.42(\mathrm{~m}, 1 \mathrm{H}), 3.21(\mathrm{dd}, J$
$=14.4,7.8 \mathrm{~Hz}, 1 \mathrm{H}), 2.94(\mathrm{dd}, J=14.4,8.5 \mathrm{~Hz}, 1 \mathrm{H}), 1.89(\mathrm{~s}, 3$ $\mathrm{H}), 1.26(\mathrm{~s}, 9 \mathrm{H}) \mathrm{ppm} .{ }^{13} \mathrm{C} \mathrm{NMR}\left(75 \mathrm{MHz}, \mathrm{CDCl}_{3}\right): \delta=169.2$ 167.3, 160.8, 136.9, 129.0, 128.6, 126.7, 98.4, 71.8, 57.7, 51.2, 44.1, 34.5, 28.6, 22.6 ppm. HRMS (EI): calcd. for $\mathrm{C}_{19} \mathrm{H}_{26} \mathrm{~N}_{2} \mathrm{O}_{3}[\mathrm{M}]^{+}$ 330.1943 ; found 330.1948

$\mathrm{N}$-tert-Butyl-4-methyl-2-[7-methyl-5-oxo-2,3-dihydro-1,4-oxazepin4(5H)-yl]pentanamide (4m): Elution with heptane/EtOAc $(50 \%)$ provided pure $4 \mathrm{~m}$, yield $84.5 \mathrm{mg}$, 95\% (procedure A); $75.6 \mathrm{mg}$, 85\% (procedure B). ${ }^{1} \mathrm{H}$ NMR $\left(300 \mathrm{MHz}, \mathrm{CDCl}_{3}\right): \delta=6.02$ (br. s, $1 \mathrm{H}$ ), $5.14(\mathrm{~s}, 1 \mathrm{H}), 4.98(\mathrm{dd}, J=8.5,6.7 \mathrm{~Hz}, 1 \mathrm{H}), 4.37(\mathrm{dd}, J=12.4$, $6.3 \mathrm{~Hz}, 1 \mathrm{H}), 4.03(\mathrm{dd}, J=12.4,6.6 \mathrm{~Hz}, 1 \mathrm{H}), 3.67(\mathrm{dd}, J=15.9$, $6.3 \mathrm{~Hz}, 1 \mathrm{H}), 3.45$ (dd, $J=15.9,6.6 \mathrm{~Hz}, 1 \mathrm{H}), 1.94(\mathrm{~s}, 3 \mathrm{H}), 1.70$ $1.35(\mathrm{~m}, 3 \mathrm{H}), 1.30(\mathrm{~s}, 9 \mathrm{H}), 0.92(\mathrm{~d}, J=6.5 \mathrm{~Hz}, 3 \mathrm{H}), 0.90(\mathrm{~d}, J=$ $6.5 \mathrm{~Hz}, 3 \mathrm{H}) \mathrm{ppm} .{ }^{13} \mathrm{C} \mathrm{NMR}\left(75 \mathrm{MHz}, \mathrm{CDCl}_{3}\right): \delta=169.8,167.2$, 160.6, 98.6, 71.9, 55.1, 51.1, 43.8, 36.8, 28.6, 25.0, 23.1, 22.5, 22.2 ppm. HRMS (ESI): calcd. for $\mathrm{C}_{16} \mathrm{H}_{29} \mathrm{~N}_{2} \mathrm{O}_{3}[\mathrm{M}+\mathrm{H}]^{+}$297.2173; found 297.2180

$\mathrm{N}$-tert-Butyl-4-butyl-7-methyl-5-oxo-2,3,4,5-tetrahydro-1,4-oxazepine-3-carboxamide (4n): Elution with heptane/EtOAc (50\%) provided pure $4 \mathbf{n}$, yield $69.5 \mathrm{mg}, 82 \%$ (procedure A); $75.4 \mathrm{mg}, 89 \%$ (procedure B). ${ }^{1} \mathrm{H}$ NMR $\left(300 \mathrm{MHz}, \mathrm{CDCl}_{3}\right): \delta=5.86$ (br. s, $1 \mathrm{H}$ ), $5.17(\mathrm{~s}, 1 \mathrm{H}), 5.01(\mathrm{dd}, J=12.0,5.4 \mathrm{~Hz}, 1 \mathrm{H}), 4.06(\mathrm{~d}, J=5.4 \mathrm{~Hz}$, $1 \mathrm{H}), 3.98(\mathrm{~d}, J=12.0 \mathrm{~Hz}, 1 \mathrm{H}), 3.69-3.52(\mathrm{~m}, 1 \mathrm{H}), 3.31-3.16(\mathrm{~m}$, $1 \mathrm{H}), 1.92(\mathrm{~s}, 3 \mathrm{H}), 1.65-1.20(\mathrm{~m}, 13 \mathrm{H}), 0.93(\mathrm{t}, J=7.2 \mathrm{~Hz}, 3 \mathrm{H})$ ppm. ${ }^{13} \mathrm{C}$ NMR $\left(75 \mathrm{MHz}, \mathrm{CDCl}_{3}\right): \delta=166.3,165.5,162.6,99.2$, $71.5,64.6,51.7,49.3,29.6,28.6,21.9,20.2,13.8$ ppm. HRMS (ESI): calcd. for $\mathrm{C}_{15} \mathrm{H}_{27} \mathrm{~N}_{2} \mathrm{O}_{3}[\mathrm{M}+\mathrm{H}]^{+}$283.2016; found 283.2019.

$N$-tert-Butyl-4-(4-methoxybenzyl)-7-methyl-5-oxo-2,3,4,5-tetrahydro-1,4-oxazepine-3-carboxamide (40): Elution with heptane/ EtOAc $(50 \%$ ) provided pure $4 \mathbf{0}$, yield $85.2 \mathrm{mg}, 82 \%$ (procedure A); $82.1 \mathrm{mg}, 79 \%$ (procedure B). ${ }^{1} \mathrm{H}$ NMR $\left(300 \mathrm{MHz}, \mathrm{CDCl}_{3}\right): \delta=$ $7.24(\mathrm{~d}, J=8.6 \mathrm{~Hz}, 2 \mathrm{H}), 6.85(\mathrm{~d}, J=8.6 \mathrm{~Hz}, 2 \mathrm{H}), 5.62$ (br. s, 1 H), $5.23(\mathrm{~s}, 1 \mathrm{H}), 4.92(\mathrm{dd}, J=12.0,5.4 \mathrm{~Hz}, 1 \mathrm{H}), 4.66(\mathrm{~d}, J=$ $14.3 \mathrm{~Hz}, 1 \mathrm{H}), 4.54(\mathrm{~d}, J=14.3 \mathrm{~Hz}, 1 \mathrm{H}), 4.08(\mathrm{~d}, J=5.4 \mathrm{~Hz}, 1$ H), $3.85(\mathrm{~d}, J=12.0 \mathrm{~Hz}, 1 \mathrm{H}), 3.79(\mathrm{~s}, 3 \mathrm{H}), 1.91(\mathrm{~s}, 3 \mathrm{H}), 1.18(\mathrm{~s}$, $9 \mathrm{H}) \mathrm{ppm} .{ }^{13} \mathrm{C}$ NMR $\left(75 \mathrm{MHz}, \mathrm{CDCl}_{3}\right): \delta=165.8,165.7,162.9$, $159.5,130.2,128.6,114.4,98.9,71.6,63.5,55.3,51.9,51.5,28.3$, 22.0 ppm. HRMS (ESI): calcd. for $\mathrm{C}_{19} \mathrm{H}_{27} \mathrm{~N}_{2} \mathrm{O}_{4}[\mathrm{M}+\mathrm{H}]^{+}$ 347.1965 ; found 347.1966

\section{Acknowledgments}

Support was provided by the Research Foundation - Flanders (FWO) and by the Research Fund of the University of Leuven (KU Leuven). A. A. P. and A. A. N. are grateful to the Erasmus Mundus External Cooperation Window (EMECW) - Triple I for providing doctoral scholarships.

[1] For selected reviews, see: a) H. Huang, Y. Zhou, H. Liu, Beilstein J. Org. Chem. 2011, 7, 897-936; b) H. C. Shen, Tetrahedron 2008, 64, 3885-3903; c) A. S. K. Hashmi, M. Rudolph, Chem. Commun. 2011, 47, 6536-6544; d) A. S. K. Hashmi, M. Bührle, Aldrichim. Acta 2010, 43, 27-33; e) X. Zeng, Chem. Rev. 2013, 113, 6864-6900; f) A. S. Dudnik, N. Chernyak, V. Gevorgyan, Aldrichim. Acta 2010, 43, 37-46; g) S. F. Kirsch, Synthesis 2008, 3183-3204; h) R. A. Widenhoefer, F. Song, in Catalyzed Carbon-Heteroatom Bond Formation (Ed.: A. K. Yudin), Wiley-VCH, Weinheim, Germany, 2010, p. 437-461; i) R. A. Widenhoefer, F. Song, in: Catalyzed Carbon-Heteroatom Bond Formation (Ed.: A. K. Yudin), Wiley-VCH, Weinheim, Germany, 2010, p. 463-492; j) P. Belmont, in: Silver in Organic Chemistry (Ed.: M. Harmata), John Wiley \& Sons, Hoboken, 2010, p. 143-165.
451 
[2] For representative examples, see: a) R. Kazem Shiroodi, C. Rivera Vera, A. S. Dudnik, V. Gevorgyan, Tetrahedron Lett. 2015 , DOI: <doi $>10.1016 /$ j.tetlet.2015.01.006</doi $>$; b) A. S. K Hashmi, T. Häffner, M. Rudolph, F. Rominger, Eur. J. Org. Chem. 2011, 667-671; c) J. P. Weyrauch, A. S. K. Hashmi, A Schuster, T. Hengst, S. Schetter, A. Littmann, M. Rudolph, M. Hamzic, J. Visus, F. Rominger, W. Frey, J. W. Bats, Chem. Eur. J. 2010, 16, 956-963; d) V. A. Peshkov, O. P. Pereshivko, S. Sharma, T. Meganathan, V. S. Parmar, D. S. Ermolat'ev, E. V Van der Eycken, J. Org. Chem. 2011, 76, 5867-5872; e) O. P. Pereshivko, V. A. Peshkov, A. A. Peshkov, J. Jacobs, L. Van Meervelt, E. V. Van der Eycken, Org. Biomol. Chem. 2014 12, 1741-1750; f) N. Sakai, N. Uchida, T. Konakahara, Tetrahedron Lett. 2008, 49, 3437-3440.
[3] For representative examples, see: a) S. G. Modha, A. Kumar, D. D. Vachhani, J. Jacobs, S. K. Sharma, V. S. Parmar, L. Van Meervelt, E. V. Van der Eycken, Angew. Chem. Int. Ed. 2012, 51, 9572-9575; Angew. Chem. 2012, 124, 9710; b) A. S. K. Hashmi, M. Rudolph, J. Huck, W. Frey, J. W. Bats, M Hamzic, Angew. Chem. Int. Ed. 2009, 48, 5848-5852; Angew. Chem. 2009, 121, 5962; c) Y. Zhou, E. Feng, G. Liu, D. Ye, J Li, H. Jiang, H. Liu, J. Org. Chem. 2009, 74, 7344-7348; d) F. Liu, Y. Yu, J. Zhang, Angew. Chem. Int. Ed. 2009, 48, 55055508; Angew. Chem. 2009, 121, 5613; e) C. Ferrer, C. H. M. Amijs, A. M. Echavarren, Chem. Eur. J. 2007, 13, 1358-1373.

[4] a) C. Ferrer, A. M. Echavarren, Angew. Chem. Int. Ed. 2006 45, 1105-1109; Angew. Chem. 2006, 118, 1123; b) A. S. K. Hashmi, W. Yang, F. Rominger, Adv. Synth. Catal. 2012, 354, 1273-1279; c) M. Gruit, A. Pews-Davtyan, M. Beller, Org. Biomol. Chem. 2011, 9, 1148-1159; d) M. Gruit, D. Michalik, A. Tillack, M. Beller, Angew. Chem. Int. Ed. 2009, 48, 7212-7216; Angew. Chem. 2009, 121, 7348; e) G. Cera, P. Crispino, M Monari, M. Bandini, Chem. Commun. 2011, 47, 7803-7805.

[5] S. G. Modha, D. D. Vachhani, J. Jacobs, L. Van Meervelt, E. V. Van der Eycken, Chem. Commun. 2012, 48, 6550-6552.

[6] A. Kumar, Z. Li, S. K. Sharma, V.S. Parmar, E. V. Van der Eycken, Chem. Commun. 2013, 49, 6803-6805.

[7] Z. Li, A. Kumar, D. D. Vachhani, S. K. Sharma, V. S. Parmar, E. V. Van der Eycken, Eur. J. Org. Chem. 2014, 2084-2091

[8] A. Kumar, Z. Li, S. K. Sharma, V. S. Parmar, E. V. Van der Eycken, Org. Lett. 2013, 15, 1874-1877.
[9] V. A. Peshkov, O. P. Pereshivko, E. V. Van der Eycken, $A d v$. Synth. Catal. 2012, 354, 2841-2848.

[10] Considering the known reactivity of hydroxyl nucleophiles in gold-catalyzed additions to $\mathrm{C}-\mathrm{C}$ triple bonds, such an outcome is not completely surprising. For representative examples, see: a) M. Schuler, F. Silva, C. Bobbio, A. Tessier, V. Gouverneur, Angew. Chem. Int. Ed. 2008, 47, 7927-7930; Angew. Chem. 2008, 120, 8045; b) R. Rüttinger, J. Leutzow, M. Wilsdorf, K. Wilckens, C. Czekelius, Org. Lett. 2011, 13, 224-227; c) K. Wilckens, M. Uhlemann, C. Czekelius, Chem. Eur. J. 2009, 15, 13323-13326; d) L.-P. Liu, G. B. Hammond, Org. Lett. 2009 , 11, 5090-5092; e) A. Aponick, C.-Y. Li, J. A. Palmes, Org. Lett. 2009, 11, 121-124; f) Y. Zhang, J. J. Xue, Z. J. Xin, Z. X. Xie, Y. Li, Synlett 2008, 940-944; g) A. Zhdanko, M. E. Maier, Eur. J. Org. Chem. 2014, 3411-3422.

[11] During the preparation of this manuscript a similar approach towards oxazocines was reported, see: S. S. Scully, S.-L. Zheng, B. K. Wagner, S. L. Schreiber, Org. Lett. 2015, 17, 418-421.

[12] For another relevant process that utilizes $\mathrm{PtCl}_{4}$ as the catalyst for the synthesis of bicyclic acetals featuring either an oxazipine or oxazocine moiety, see: A. Diéguez-Vázquez, C. C. Tzschucke, W. Y. Lam, S. V. Ley, Angew. Chem. Int. Ed. 2008, 47, 209-212; Angew. Chem. 2008, 120, 216.

[13] For reviews focusing on the synthesis of heterocycles by the Ugi reaction followed by secondary transformations, see: a) L. Banfi, A. Basso, R. Riva, Top. Heterocycl. Chem. 2010, 23, 139; b) U. K. Sharma, N. Sharma, D. D. Vachhani, E. V. Van der Eycken, Chem. Soc. Rev. 2015, 44, 1836-1860.

[14] For recent applications of the Ugi reaction to the synthesis of medium-ring heterocycles, see: a) S. Pandey, S. V. Kumar, R. Kant, P. M. S. Chauhan, Org. Biomol. Chem. 2014, 12, 5346 5350; b) D. D. Vachhani, A. Kumar, S. G. Modha, S. K. Sharma, V. S. Parmar, E. V. Van der Eycken, Eur. J. Org. Chem. 2013, 1223-1227; c) Z. Li, L. Legras, A. Kumar, D. D. Vachhani, S. K. Sharma, V. S. Parmar, E. V. Van der Eycken, Tetrahedron Lett. 2014, 55, 2070-2074; d) L. Moni, L. Banfi, A. Basso, A. Brambilla, R. Riva, Beilstein J. Org. Chem. 2014, 10, 209-212; e) A. A. Peshkov, V. A. Peshkov, O. P. Pereshivko, E. V. Van der Eycken, 2015, DOI: 10.1016/j.tet.2015.04.022.

Received: March 17, 2015 
591

$$
\begin{aligned}
& \mathrm{A}=5 \mathrm{~mol}-\% \mathrm{AuPPh}_{3} \mathrm{Cl} / \mathrm{AgOTf}, 2 \mathrm{~h}, 25^{\circ} \mathrm{C} \\
& \mathrm{B}=5 \mathrm{~mol}-\% \mathrm{AgOTf}^{\circ} 15 \mathrm{~h}, 50^{\circ} \mathrm{C}
\end{aligned}
$$

A comprehensive study on gold- and silvercatalyzed cyclisomerizations of hydroxy- propargylamides into oxazepines is described.
A. A. Peshkov, A. A. Nechaev,

O. P. Pereshivko, J. L. Goeman,

J. Van der Eycken, V. A. Peshkov,*

E. V. Van der Eycken*

1-9

Gold- and Silver-Catalyzed 7-endo-dig Cyclizations for the Synthesis of Oxazepines

Keywords: Synthetic methods / Multicomponent reactions / Medium-ring compounds / Cyclization / Nitrogen heterocycles 